\title{
Noncoherent Spectral Optical CDMA System Using 1D Active Weight Two-Code Keying Codes
}

\author{
Bih-Chyun Yeh \\ Department of Electrical Engineering, School of Electrical and Computer Engineering, College of Engineering, \\ Chang-Gung University, Tao-Yuan, Taiwan \\ Correspondence should be addressed to Bih-Chyun Yeh; bih.chyun.yeh@gmail.com
}

Received 5 March 2016; Accepted 4 October 2016

Academic Editor: Antonino Laudani

Copyright (C) 2016 Bih-Chyun Yeh. This is an open access article distributed under the Creative Commons Attribution License, which permits unrestricted use, distribution, and reproduction in any medium, provided the original work is properly cited.

\begin{abstract}
We propose a new family of one-dimensional (1D) active weight two-code keying (TCK) in spectral amplitude coding (SAC) optical code division multiple access (OCDMA) networks. We use encoding and decoding transfer functions to operate the 1D active weight TCK. The proposed structure includes an optical line terminal (OLT) and optical network units (ONUs) to produce the encoding and decoding codes of the proposed OLT and ONUs, respectively. The proposed ONU uses the modified cross-correlation to remove interferences from other simultaneous users, that is, the multiuser interference (MUI). When the phase-induced intensity noise (PIIN) is the most important noise, the modified cross-correlation suppresses the PIIN. In the numerical results, we find that the bit error rate (BER) for the proposed system using the 1D active weight TCK codes outperforms that for two other systems using the $1 \mathrm{D}$ M-Seq codes and 1D balanced incomplete block design (BIBD) codes. The effective source power for the proposed system can achieve $-10 \mathrm{dBm}$, which has less power than that for the other systems.
\end{abstract}

\section{Introduction}

Optical code division multiple access (OCDMA) systems are used in various network applications and their performance has become crucial [1-4]. These systems have the potential to provide network control, enhanced information security, and improved spectral efficiency [5-8]. OCDMA systems use a passive optical network (PON), which requires optical components such as couplers, splitters, combiners, and switches, and use multiple access points to remove interferences in the code sequences that are caused by different users. Encoding/decoding approaches are used to construct OCDMA systems and suppress the multiuser interference (MUI) [9-11] that arises when using shared channels with the same frequency spectrum. In addition, the OCDMA systems use photodiodes to shield them from the effects of phaseinduced intensity noise (PIIN) [12-16], a design feature of such systems.

Seyedzadeh et al. [17] reported experimental and simulation results from a variable-weight spectral amplitude coding optical code division multiple access (VW-SACOCDMA) system. The results outlined a back-to-back system with a minimum average power per chip, which maintained acceptable performance. The transmission used $60 \mathrm{~km}$ of fiber. The VW-SAC-OCDMA system used a mathematical approximation to calculate its capacity. The experimental results showed the bit error rate (BER) in the channels. The system uses the VW-SAC-OCDMA as a potential solution for QoS differentiation and flexible structure in metro networks.

Mostafa et al. [18] proposed that the PIIN and MUI are the main parameters that affect the performance of OCDMA systems. They lead to degradations in system performance and a reduction in the number of active users. The authors presented a system with an efficiently modified multiservice (MMS) code to produce the encoding spectral amplitude of the optical source in OCDMA systems, which avoided the PIIN and MUI. The code disposed the effect of the MUI and PIIN and gave better performance than other traditional codes. Also, the system had a practical code length with a simple receiver structure. The design of the codes in the SACOCDMA system used a critical parameter to overcome the system's drawbacks. Yeh [19] proposed the 1D added length codes to add the code length. At the same time, the crosscorrelation of the $1 \mathrm{D}$ added length codes eliminated the 
MUI and suppressed the PIIN. The 1D added length codes produced the scheme using one-code keying.

In this paper, we propose a new family of new codes for OCDMA systems named 1D active weight two-code keying (TCK) codes. We use an encoding transfer function to transfer the information bits into the code sequences of the $1 \mathrm{D}$ active weight TCK codes and a decoding transfer function to transfer the code sequences into the recovered bits. The optical line terminal (OLT) of the proposed structure obtains the information bits used to produce the encoding codes, and the optical network units (ONUs) of the proposed structure obtain the code sequences used to produce the decoding codes. The proposed ONUs use the modified crosscorrelation to calculate the recovered bits. Because the photodiodes suffer from interference from other simultaneous users (i.e., MUI), the modified cross-correlation removes this. The PIIN is the most prominent type of noise and is based on the number of simultaneous users; the proposed ONUs use the modified cross-correlation to suppress the PIIN. In the numerical results, with an effective source power of $-10 \mathrm{dBm}$, a data transmission rate of $2.5 \mathrm{Gbps}$, and a BER of $10^{-9}$, the number of simultaneous users for the proposed system is 30 users. The number of possible simultaneous users for the proposed system is larger than that for other systems, that is, those that use $1 \mathrm{D}$ M-Seq codes and $1 \mathrm{D}$ BIBD codes. The effective source power for the proposed system using the 1D active weight TCK codes is $-10 \mathrm{dBm}$, which is lower power than that for the other systems.

The rest of the paper is organized as follows. The properties of the 1D active weight TCK codes are derived in Section 2. In Section 3, we describe the system architecture. In Section 4, we analyze the system performance using the photocurrents of the photodiodes to determine the signal to noise ratio (SNR) and the BER. In Section 5, we show the numerical results of the performance of the $1 \mathrm{D}$ active weight TCK codes. Finally, in Section 6, we provide summary and conclusions.

\section{1D Active Weight TCK Codes}

We propose one-dimensional (1D) active weight TCK codes for use in spectral amplitude coding (SAC) OCDMA networks. In this paper, we introduce a code family to create the codes, which produces their code sequences. The codes use two-code keying to generate the code sequences, which are defined by the information bits " 0 " and " 1 ." These code sequences are used by optical communication systems. The proposed codes generate a Galois field of $\mathrm{GF}(k)$, where $k$ is a prime number. The codes have a weight of $k$ and a length of $2 k^{2}$, and an encoding transfer function is used to produce the code weight position. The code weight position is a function of the prime number $k$ and is defined as follows:

$$
S_{M, O, m}(p)=2(p * M-k-O-r) \quad \bmod k+m-1,
$$

where $k$ is a prime number with the Galois field $\mathrm{GF}(k), p$ is $[0,1, \ldots, k-1], M$ is $[0,1, \ldots, k-1], O$ is $[0,1, \ldots, k-1], r$ is a fixed value in the range $[0,1, \ldots, k-1]$, mod is the remainder calculation for the modulo operation, and $m$ is $[1,2]$. The code weight position is directly converted to $[0,1, \ldots, 2 k-1]$.
We use the code weight position to extend the code sequences $c_{M, O, m}(n)$ as follows:

$$
c_{M, O, m}(n)= \begin{cases}1, & \text { if } n=2 k p+S_{M, O, m}(p) \\ 0, & \text { else }\end{cases}
$$

where $n$ is the input of $c_{M, O, m}(n)$ and $c_{M, O, m}(n)$ is the elements of the code sequence $C_{M, O, m}$ of the codes. Table 1 shows the codes derived using the prime number $k=3$.

The proposed codes use the derived code sequences to operate the decoding transfer function, which produces a modified cross-correlation. The cross-correlation between the code sequences $C_{M, O, m}$ and $C_{M^{\prime}, \mathrm{O}^{\prime}, m^{\prime}}$ is expressed as follows:

$$
C_{M, O, m} \odot C_{M^{\prime}, \mathrm{O}^{\prime}, m^{\prime}}=\sum_{n=0}^{2 k^{2}-1} c_{M, O, m}(n) c_{M^{\prime}, \mathrm{O}^{\prime}, m^{\prime}}(n),
$$

where the code sequences $C_{M, \mathrm{O}, m}$ and $C_{M^{\prime}, \mathrm{O}^{\prime}, m^{\prime}}$ are both $1 \mathrm{D}$ active weight TCK codes, $\odot$ is the dot-product of two vectors, and $c_{M, O, m}(n)$ and $c_{M^{\prime}, \mathrm{O}^{\prime}, m^{\prime}}(n)$ are the elements of the code sequences $C_{M, O, m}$ and $C_{M^{\prime}, \mathrm{O}^{\prime}, m^{\prime}}$, respectively. The crosscorrelation of the codes is expressed as follows:

$$
\begin{aligned}
& C_{M, O, m} \odot C_{M^{\prime}, O^{\prime}, m^{\prime}} \\
& \quad= \begin{cases}k, & M=M^{\prime}, \quad O=O^{\prime}, \quad m=m^{\prime} \\
0, & M=M^{\prime}, \quad O \neq O^{\prime}, \quad m=m^{\prime} \text { or } m \neq m^{\prime} \\
1, & M \neq M^{\prime}, \quad m=m^{\prime},\end{cases}
\end{aligned}
$$

where $k$ is the code weight. The OCDMA system uses the code sequences to create cross-correlations exactly equal to $k, 0$, and 1 . As shown in equation (4), the cross-correlations of the $1 \mathrm{D}$ active weight TCK codes are $k$ for $M=M^{\prime}, O=O^{\prime}$, $m=m^{\prime}, 0$ for $M=M^{\prime}, O \neq O^{\prime}, m=m^{\prime}$ or $m \neq m^{\prime}$, and 1 for $M \neq M^{\prime}, m=m^{\prime}$; that is, the codes have an autocorrelation value of $k$ and have cross-correlation values of 0 or 1 . When the proposed codes use cross-correlation, this causes interference in the code sequences.

The code sequence of the 1D active weight TCK codes has the one-characteristic matrix $C_{M, O+1, m}$. We generate the code sequence $C_{M, O+1, m}$ to produce the cross-correlation between the code sequences $C_{M, O+1, m}$ and $C_{M^{\prime}, \mathrm{O}^{\prime}, m^{\prime}}$ as follows:

$$
\begin{aligned}
& C_{M, O+1, m} \odot C_{M^{\prime}, \mathrm{O}^{\prime}, m^{\prime}} \\
& \quad= \begin{cases}k, & M=M^{\prime}, O+1=O^{\prime}, m=m^{\prime} \\
0, & M=M^{\prime}, O+1 \neq O^{\prime}, m=m^{\prime} \text { or } m \neq m^{\prime} \\
1, & M \neq M^{\prime}, \quad m=m^{\prime} .\end{cases}
\end{aligned}
$$

Thus, $M=M^{\prime}, O+1=O^{\prime}$, and $m=m^{\prime}$ produce a crosscorrelation $=k, M=M^{\prime}, O+1 \neq O^{\prime}, m=m^{\prime}$, and $m \neq m^{\prime}$ produce a cross-correlation $=0$, and $M \neq M^{\prime}$, $m=m^{\prime}$ produce a cross-correlation $=1$. We use $C_{M, O, m} \odot$ 
TABLE 1: Code sequences of the 1D active weight TCK codes with $k=3$.

\begin{tabular}{llllllllllllllllllll}
\hline \multicolumn{11}{c}{ Code sequences of the 1D active weight TCK codes } \\
\hline 0 & 0 & 0 & 0 & 1 & 0 & 0 & 0 & 0 & 0 & 1 & 0 & 0 & 0 & 0 & 0 & 1 & 0 \\
0 & 0 & 1 & 0 & 0 & 0 & 0 & 0 & 1 & 0 & 0 & 0 & 0 & 0 & 1 & 0 & 0 & 0 \\
1 & 0 & 0 & 0 & 0 & 0 & 1 & 0 & 0 & 0 & 0 & 0 & 1 & 0 & 0 & 0 & 0 & 0 \\
0 & 0 & 0 & 0 & 1 & 0 & 1 & 0 & 0 & 0 & 0 & 0 & 0 & 0 & 1 & 0 & 0 & 0 \\
0 & 0 & 1 & 0 & 0 & 0 & 0 & 0 & 0 & 0 & 1 & 0 & 1 & 0 & 0 & 0 & 0 & 0 \\
1 & 0 & 0 & 0 & 0 & 0 & 0 & 0 & 1 & 0 & 0 & 0 & 0 & 0 & 0 & 0 & 1 & 0 \\
0 & 0 & 0 & 0 & 1 & 0 & 0 & 0 & 1 & 0 & 0 & 0 & 1 & 0 & 0 & 0 & 0 & 0 \\
0 & 0 & 1 & 0 & 0 & 0 & 1 & 0 & 0 & 0 & 0 & 0 & 0 & 0 & 0 & 0 & 1 & 0 \\
1 & 0 & 0 & 0 & 0 & 0 & 0 & 0 & 0 & 0 & 1 & 0 & 0 & 0 & 1 & 0 & 0 & 0 \\
0 & 0 & 0 & 0 & 0 & 1 & 0 & 0 & 0 & 0 & 0 & 1 & 0 & 0 & 0 & 0 & 0 & 1 \\
0 & 0 & 0 & 1 & 0 & 0 & 0 & 0 & 0 & 1 & 0 & 0 & 0 & 0 & 0 & 1 & 0 & 0 \\
0 & 1 & 0 & 0 & 0 & 0 & 0 & 1 & 0 & 0 & 0 & 0 & 0 & 1 & 0 & 0 & 0 & 0 \\
0 & 0 & 0 & 0 & 0 & 1 & 0 & 1 & 0 & 0 & 0 & 0 & 0 & 0 & 0 & 1 & 0 & 0 \\
0 & 0 & 0 & 1 & 0 & 0 & 0 & 0 & 0 & 0 & 0 & 1 & 0 & 1 & 0 & 0 & 0 & 0 \\
0 & 1 & 0 & 0 & 0 & 0 & 0 & 0 & 0 & 1 & 0 & 0 & 0 & 0 & 0 & 0 & 0 & 1 \\
0 & 0 & 0 & 0 & 0 & 1 & 0 & 0 & 0 & 1 & 0 & 0 & 0 & 1 & 0 & 0 & 0 & 0 \\
0 & 0 & 0 & 1 & 0 & 0 & 0 & 1 & 0 & 0 & 0 & 0 & 0 & 0 & 0 & 0 & 0 & 1 \\
0 & 1 & 0 & 0 & 0 & 0 & 0 & 0 & 0 & 0 & 0 & 1 & 0 & 0 & 0 & 1 & 0 & 0 \\
\hline
\end{tabular}

$C_{M^{\prime}, \mathrm{O}^{\prime}, m^{\prime}}$ and $C_{M, \mathrm{O}+1, m} \odot C_{M^{\prime}, \mathrm{O}^{\prime}, m^{\prime}}$ to produce the modified cross-correlation using the following transformation:

$$
\begin{aligned}
& C_{M, O, m} \odot C_{M^{\prime}, O^{\prime}, m^{\prime}}-C_{M, O+1, m} \odot C_{M^{\prime}, O^{\prime}, m^{\prime}} \\
& \quad= \begin{cases}k, & M=M^{\prime}, O=O^{\prime}, m=m^{\prime}, \\
-k, & M=M^{\prime}, O+1=O^{\prime}, m=m^{\prime}, \\
0, & \text { otherwise, }\end{cases}
\end{aligned}
$$

where the values of the modified cross-correlation are the ideal cross-correlation with $k,-k$, and 0 , respectively. Furthermore, we subtract $C_{M, \mathrm{O}+1, m} \odot C_{M^{\prime}, \mathrm{O}^{\prime}, m^{\prime}}$ from $C_{M, \mathrm{O}, m} \odot$ $C_{M^{\prime}, O^{\prime}, m^{\prime}}$ to produce the modified cross-correlation. The proposed codes use the modified cross-correlation $=k$ for $M=M^{\prime}, O=O^{\prime}, m=m^{\prime}$ to produce the code sequence $C_{M, O, m}$ from the recovered "0" bits. Similarly, the proposed codes use the modified cross-correlation $=-k$ for $M=M^{\prime}, O+1=O^{\prime}, m=m^{\prime}$ to produce the code sequence $C_{M, O+1, m}$ from the recovered " 1 " bits. The proposed codes use the modified cross-correlation $=0$ otherwise. Therefore, the proposed codes produce the code sequences $C_{M, \mathrm{O}, m}$ and $C_{M, \mathrm{O}+1, m}$, which are the recovered "0" and "1" bits, respectively.

First, cardinality comparison, the 1D active weight TCK codes get the code length equal to code size to produce the better cardinality. Then, the $1 \mathrm{D}$ MDW codes proposed by Aljunid et al. [20] used the code length to reduce the code size, which made the cardinality loss. The cardinality of the $1 \mathrm{D}$ active weight TCK codes is better than that of the $1 \mathrm{D}$ MDW codes.

Second, in autocorrelation and cross-correlation comparison, we find the $1 \mathrm{D}$ MDW codes with the one-code key
(OCK) to get the autocorrelation and cross-correlation [20]. The 1D MDW codes get the cross-correlation of the 1D MDW codes to produce the OCK. In fact, the comparison between and $1 \mathrm{D}$ active weight TCK codes and 1D MDW codes with the OCK is dependent on the cross-correlation. The $1 \mathrm{D}$ active weight TCK codes transfer the information bit $=$ " 1 " and " 0 " into the code sequence with the information bit " 1 " and code sequence with the information bit " 0 ," which get " $k$ " of the cross-correlation in the receiver and " $-k$ " of the crosscorrelation in the receiver to combine the level " 0 " of the cross-correlation in the receiver. The 1D MDW codes transfer the information bit $=$ " 1 " and " 0 " into the code sequence with the information bit " 1 " and no code sequence, which get " $k$ " of the cross-correlation in the receiver and " 0 " of the cross-correlation in the receiver to combine the level " $k / 2$ " of the cross-correlation in the receiver. The level of the cross-correlation in the receiver in the $1 \mathrm{D}$ active weight TCK codes shows better performance than that in the ID MDW codes.

We get the first cardinality comparison and second autocorrelation and cross-correlation comparison to operate the method comparison. The method comparison of the 1D active weight TCK codes is better than that of the 1D MDW codes.

Furthermore, when the code sequences $C_{M, \mathrm{O}, m}$ and $C_{M, O+1, m}$ are assigned to the same user for two-code keying, we use a MUI elimination scheme to solve the problem of interference from other simultaneous users using the modified cross-correlation. When we use the $1 \mathrm{D}$ active weight TCK codes to produce the modified cross-correlation, the modified cross-correlation produces a recovered " 0 " or "1" bit. Moreover, photodiodes using the modified crosscorrelation suppress the PIIN. Therefore, the proposed codes 


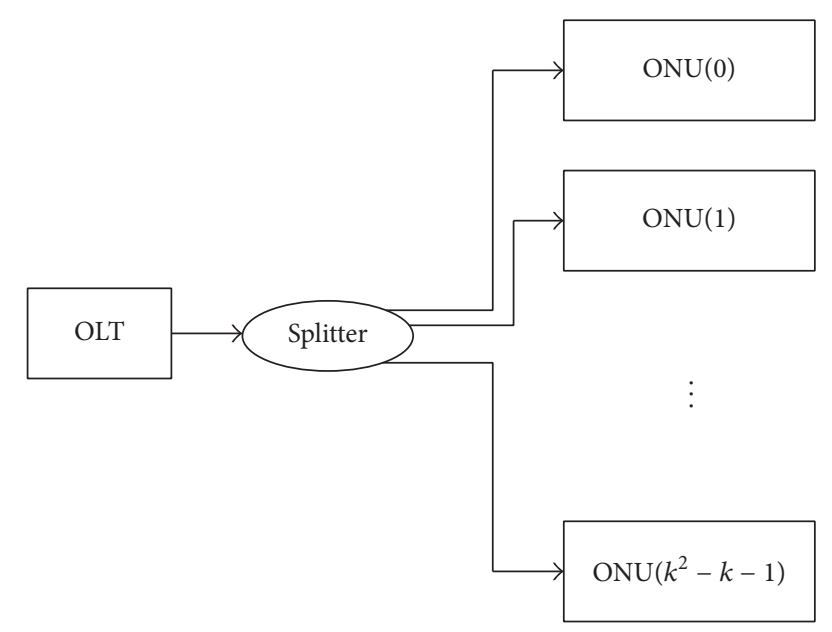

FIgURE 1: Schematic block diagram of the 1D active weight TCK codes.

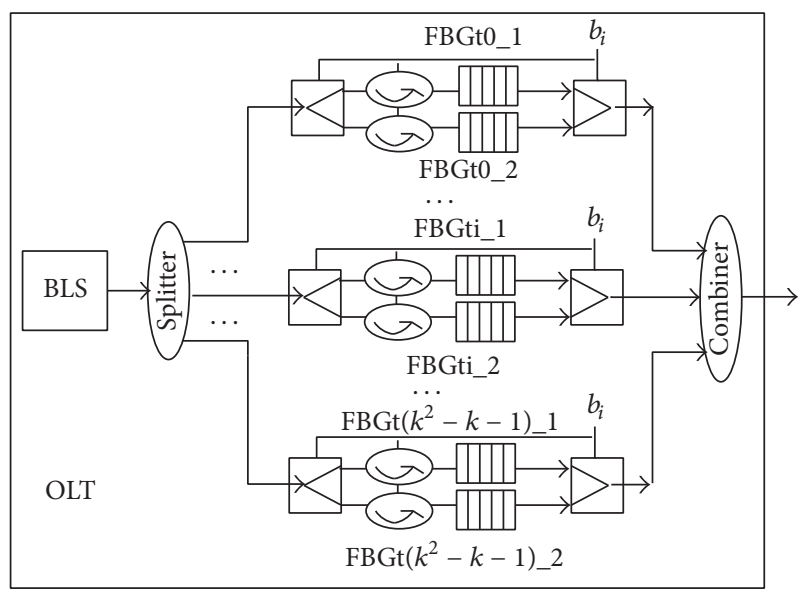

FIgURE 2: The structure of the OLT.

use the modified cross-correlation to remove MUI and suppress the PIIN.

\section{System Description}

Figure 1 shows the schematic block diagram of the 1D active weight TCK codes. The proposed structure of the OCDMA system comprises the OLT, a $\left(k^{2}-k\right)$ optical splitter, and $\left(k^{2}-k\right)$ ONUs. The optical signals using the code sequences are sent from the OLT to the optical splitter, which connects to the ONUs. The optical splitter sends the code sequences into the ONUs, each of which recovers the appropriate bits from the code sequences. We use the proposed structure of the OCDMA system to develop the OLT, the optical splitter, and the ONUs.

Figure 2 shows the proposed structure of the OLT, which comprises an unpolarized broadband light source (BLS), a $\left(k^{2}-k\right)$ optical splitter, $\left(k^{2}-k\right) 1 \times 2$ optical switches with controlled value $b_{i}, i=0,1, \ldots,\left(k^{2}-k\right)-1,2 *\left(k^{2}-k\right)$ optical circulators, $2 *\left(k^{2}-k\right)$ fiber Bragg gratings, $\left(k^{2}-k\right) 2 \times 1$ optical

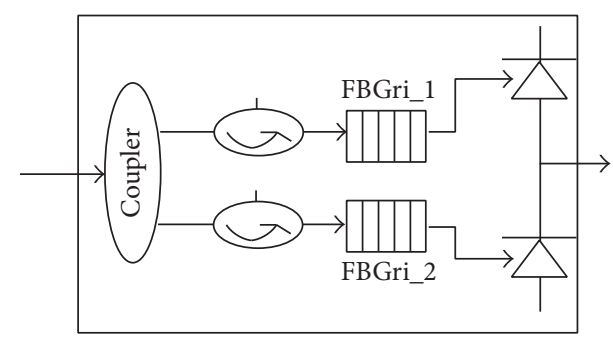

Figure 3: The structure of the ONU(i).

switches with controlled value $b_{i}, i=0,1, \ldots,\left(k^{2}-k\right)-1$, and a $\left(k^{2}-k\right) \times 1$ optical combiner. The unpolarized BLS connects to the optical splitter, which connects to the $1 \times 2$ optical switches. These switches then connect to the optical circulators, which connect to the fiber Bragg gratings and then to the $2 \times 1$ optical switches. Finally, these switches connect to the optical combiner.

The unpolarized BLS emits light, which passes through the optical splitter and is controlled by the value $b_{i}$. If the information bit $b_{i}=0$, the light is switched to the optical circulator and fiber Bragg grating FBGti_l. The optical signal then goes through the $2 \times 1$ switch with the control value $b_{i}=$ 0 . If the information bit $b_{i}=1$, the light is switched to the optical circulator and fiber Bragg grating FBGti_2, and the optical signal goes through the $2 \times 1$ switch with the control value $b_{i}=1$.

The fiber Bragg grating FBGti_1 corresponds to the complementary code sequence $C_{M, O, m}$ of the $1 \mathrm{D}$ active weight TCK codes; it allows the code sequence $C_{M, O, m}$ through and filters out the complementary code sequence $C_{M, O, m}$. Similarly, the FBGti_2 grating corresponds to the complementary code sequence $C_{M, O+1, m}$; it allows the code sequence $C_{M, O+1, m}$ through and filters out the complementary code sequence $C_{M, O+1, m}$.

We use the control value $b_{i}$ to allow either the fiber Bragg grating FBGti_1 or the fiber Bragg grating FBGti_2 to connect to the $2 \times 1$ optical switches. The light passing through these switches is combined by the optical combiner. Therefore, we use the information bit $b_{i}=0$ or bit $b_{i}=1$ to divert the light into either FBGti_1 or FBGti_2, which outputs code sequences with information bit 0 or information bit 1 , respectively.

Figure 3 shows the proposed structure of the $\mathrm{ONU}(i)$, $i=\left[0,1, \ldots,\left(k^{2}-k\right)-1\right]$. The structure comprises a $1 \times 2$ optical coupler, an upper optical circulator, a lower optical circulator, a fiber Bragg grating FBGri_1, a fiber Bragg grating FBGri_2, and a balanced detector between the upper and lower photodiodes. The code sequences of the $1 \mathrm{D}$ active weight TCK codes connect to the optical coupler, which connects to the upper and lower optical circulators. The upper and lower optical circulators connect to the fiber Bragg gratings FBGri_1 and FBGri_2, respectively, which then connect to the upper and lower photodiodes.

The code sequences pass through the upper optical circulator to the fiber Bragg grating FBGri_l, which corresponds to the complementary code sequence $C_{M, O, m}$. The grating FBGri_l allows the code sequence $C_{M, \mathrm{O}, m}$ through and filters out the complementary code sequence 
$C_{M, O, m}$. Similarly, in the lower optical circulator, the FBGri_2 grating allows $C_{M, O+1, m}$ code sequence through and filters out the complementary code sequence $C_{M, O+1, m}$. Thus, we use the fiber Bragg gratings FBGri_1 and FBGri_2 to send data to the upper and lower photodiodes of the balanced detector. The modified cross-correlation allows the balanced detector to obtain a recovered " 0 " or " 1 " bit.

The output photocurrent of photodiode PD0 is proportional to $C_{M, O, m} \odot C_{M^{\prime}, O^{\prime}, m^{\prime}}$, and that of photodiode PD1 is proportional to $C_{M, O+1, m} \odot C_{M^{\prime}, O^{\prime}, m^{\prime}}$. As the proposed system can be described by (6), the modified photocurrent of the modified cross-correlation is proportional to $C_{M, O, m} \odot$ $C_{M^{\prime}, \mathrm{O}^{\prime}, m^{\prime}}-C_{M, \mathrm{O}+1, m} \odot C_{M^{\prime}, \mathrm{O}^{\prime}, m^{\prime}}$, which is $k$ for $M=M^{\prime}$, $O=O^{\prime}, m=m^{\prime},-k$ for $M=M^{\prime}, O+1=O^{\prime}, m=m^{\prime}$, or 0 otherwise. Because the MUI is produced by the interferences from other simultaneous users using unmatched code words, we can use the modified cross-correlation to completely eliminate it. Moreover, because the PIIN is based on the noise in the photodiodes, it is suppressed by the photocurrent arising from the modified cross-correlation.

\section{Performance Analysis}

To analyze performance, we used the optical signal and various noise parameters to determine the SNR and BER. The optical signal is measured from the photocurrent produced by the photodiodes. The noises arise from the PIIN, shot noise, and thermal noise and change the photocurrent noise variances accordingly. The SNR is created by dividing the modified photocurrent of the optical signal by the photocurrent noises variances in the photodiodes. The BER uses the Gaussian approximation to calculate the SNR. Therefore, we can obtain the BER of the $1 \mathrm{D}$ active weight TCK codes to observe their performance.

The ONU receives the code sequences of the $1 \mathrm{D}$ active weight TCK codes, and we use the optical signals to produce the PSD of the received optical signals. The PSD of the received optical laser signals in the $\mathrm{ONU}(i)$ can be set as follows:

$$
\begin{aligned}
& R(f)=\frac{P_{\mathrm{sr}}}{\Delta f} \int_{0}^{\infty} \sum_{p=1}^{P} \Lambda(p) \\
& \quad \cdot \sum_{M^{\prime}=0}^{k-1} \sum_{O^{\prime}=0}^{k-1} \sum_{m^{\prime}=1}^{2} \sum_{q=0}^{2 k^{2}-1}\left(c_{M^{\prime}, O^{\prime}, m^{\prime}}(q)\right) F(f, q) d f
\end{aligned}
$$

where $P_{\text {sr }}$ is the effective source power at the $\mathrm{ONU}(i), \Delta f$ is the bandwidth of the source, $\Lambda(p)$ is the recovered bit of the $p$ th user, which can be 0 or $1, P$ is the number of simultaneous users, $k$ is the code weight, $c_{M^{\prime}, O^{\prime}, m^{\prime}}$ is an element of the $p$ th user's code sequence, and $F(f, q)$ is the wavelength chip. For the convenience of analysis, the proposed system defines $F(f, q)$ to be as follows:

$$
\begin{gathered}
F(f, q)=\left\{\Theta\left(f-f_{o}-\frac{\Delta f\left(-\left(2 k^{2}\right)+2 q\right) / 2}{\left(2 k^{2}\right)}\right)\right. \\
\left.-\Theta\left(f-f_{o}-\frac{\Delta f\left(-\left(2 k^{2}\right)+2 q+2\right) / 2}{\left(2 k^{2}\right)}\right)\right\} .
\end{gathered}
$$

The unit step function $\Theta(f)$ is as follows:

$$
\Theta(f)= \begin{cases}1, & f \geq 0, \\ 0, & f<0 .\end{cases}
$$

Therefore, the PSDs of the received optical signals show the power required using the code sequences to arrive at the ONUs. Based on this simplified analysis, some assumptions follow. The proposed system assumes each of the spectral components to have identical spectral width. The broadband light source is ideally unpolarized and has a flat spectrum over $\left[f_{o}-\Delta f / 2, f_{o}+\Delta f / 2\right]$, where $f_{o}$ and $\Delta f$ are the central frequency and bandwidth of the source. The proposed system thus has equal power at the ONUs.

The ONU computes a cross-correlation between the code sequences $c_{M, O, m}$ and $c_{M^{\prime}, \mathrm{O}^{\prime}, m^{\prime}}$ and between the code sequences $c_{M, O+1, m}$ and $c_{M^{\prime}, \mathrm{O}^{\prime}, m^{\prime}}$. The PSDs at the photodiodes using the cross-correlations during a one-bit period can be described as follows:

$$
\begin{aligned}
& X_{0}(f)=\frac{P_{\mathrm{sr}}}{\Delta f} \int_{0}^{\infty} \sum_{p=1}^{P} \Lambda(p) \\
& \cdot \sum_{M^{\prime}=0}^{k-1} \sum_{\mathrm{O}^{\prime}=0}^{k-1} \sum_{m^{\prime}=1}^{2} \sum_{q=0}^{2 k^{2}-1}\left(c_{M, O, m}(q) c_{M^{\prime}, O^{\prime}, m^{\prime}}(q)\right) F(f, q) d f, \\
& X_{1}(f)=\frac{P_{\mathrm{sr}}}{\Delta f} \int_{0}^{\infty} \sum_{p=1}^{P} \Lambda(p) \\
& \quad \cdot \sum_{M^{\prime}=0}^{k-1} \sum_{O^{\prime}=0}^{k-1} \sum_{m^{\prime}=1}^{2} \sum_{q=0}^{2 k^{2}-1}\left(c_{M, O+1, m}(q) c_{M^{\prime}, O^{\prime}, m^{\prime}}(q)\right) F(f, q) d f,
\end{aligned}
$$

where $k$ is the code weight of the code sequence. In addition, the proposed system uses $\Lambda(p)=1$ and $p=1,2, \ldots, P$ in the worst case, as well as the cross-correlations, to determine the maximum interferences produced by the other simultaneous users, known as the MUI. For the photodiodes PD0 and PD1, the photocurrents $I_{\mathrm{PD} 0}$ and $I_{\mathrm{PD} 1}$ are described as follows:

$$
\begin{aligned}
I_{\mathrm{PD} 0} & =R \int_{0}^{\infty} X_{0}(f) d f=R \frac{P_{\mathrm{sr}}}{\Delta f} \int_{0}^{\infty} \sum_{p=1}^{P} \Lambda(p) \\
& \cdot \sum_{M^{\prime}=0}^{k-1} \sum_{\mathrm{O}^{\prime}=0}^{k-1} \sum_{m^{\prime}=1}^{2} \sum_{q=0}^{2 k^{2}-1}\left(c_{M, \mathrm{O}, m}(q) c_{M^{\prime}, \mathrm{O}^{\prime}, m^{\prime}}(q)\right) F(f, q) d f \\
& =R \frac{P_{\mathrm{sr}}}{2 k^{2}}\left(k+\frac{(P-1) *\left(k^{2}-2 k+1\right)}{2 k^{2}-2 k-1}\right) \\
I_{\mathrm{PD} 1} & =R \int_{0}^{\infty} X_{1}(f) d f=R \frac{P_{\mathrm{sr}}}{\Delta f} \int_{0}^{\infty} \sum_{p=1}^{P} \Lambda(p) \\
& \cdot \sum_{M^{\prime}=0}^{k-1} \sum_{O^{\prime}=0}^{k-1} \sum_{m^{\prime}=1}^{2} \sum_{q=0}^{2 k^{2}-1}\left(c_{M, O+1, m}(q) c_{M^{\prime}, O^{\prime}, m^{\prime}}(q)\right) F(f, q) d f \\
= & R \frac{P_{\mathrm{sr}}}{2 k^{2}}\left(\frac{(P-1) *\left(k^{2}-2 k+1\right)}{2 k^{2}-2 k-1}\right),
\end{aligned}
$$


where $G$ is the average gain, $R$ is the responsivity of the photodiode given by $R=\eta e \lambda_{o} / h c, \eta$ is the quantum efficiency of the photodiode, $e$ is the charge on the electron, $\lambda_{o}$ is a central wavelength, $h$ is Planck's constant, $c$ is the speed of light, and $P_{\text {sr }}$ is the effective source power of each ONU. Therefore, the proposed system forces the photocurrents of the photodiodes PD0 and PD1 to be proportional to the cross-correlations and uses the cross-correlations to produce a modified photocurrent. The modified photocurrent can be written as

$$
\begin{aligned}
I_{m, \mathrm{bit}=0} & =R \int_{0}^{\infty}\left[X_{0}(f)-X_{1}(f)\right] d f=\left(I_{\mathrm{PD} 0}-I_{\mathrm{PD} 1}\right) \\
& =R \frac{P_{\mathrm{sr}}}{2 k} . \\
I_{m, \mathrm{bit}=1} & =\left(I_{\mathrm{PD} 0}-I_{\mathrm{PD} 1}\right)=-R \frac{P_{\mathrm{sr}}}{2 k} .
\end{aligned}
$$

Therefore, the proposed system uses the modified crosscorrelation to produce a modified photocurrent, removing the MUI.

The noise variances in the modified photocurrent have various effects on system performance. The photocurrent noise variances are as follows:

$$
\begin{aligned}
\left\langle i_{\text {noise }}^{2}\right\rangle & =\left\langle i_{\text {PIIN }, \mathrm{bit}=\times}^{2}\right\rangle+\left\langle i_{\text {shot,bit }=\times}^{2}\right\rangle+\left\langle i_{\text {thermal }}^{2}\right\rangle \\
& =I_{m, \mathrm{bit}=\times}^{2} B_{r} \tau+2 e I_{t, \mathrm{bit}=\times} B_{r}+\frac{4 K_{a} T_{n} B_{r}}{R_{o}},
\end{aligned}
$$

where bit $=x$ is from the " 0 " or " 1 ", PIIN, shot noise, and thermal noise are considered in the photocurrent analysis, the effect of the dark current is neglected, $I_{m, \mathrm{bit}=x}$ is the modified photocurrent, $I_{t, \mathrm{bit}=\times}$ is the total photocurrent, $B_{r}$ is the electrical bandwidth, $\tau$ is the coherence time of the light incident to the photodiode, $e$ is the charge on the electron, $K_{a}$ is Boltzmann's constant, $T_{n}$ is the absolute noise temperature, and $R_{o}$ is the load resistance. Therefore, $\tau$ can be described as follows:

$$
\tau=\frac{\int_{0}^{\infty} X^{2}(f) d f}{\left[\int_{0}^{\infty} X(f) d f\right]^{2}},
$$

where $X(f)$ is the single sideband power spectral density (PSD) of the light incident on the photodiodes. The photocurrent noise variances are based on statistically independent noise characteristics, because the PIIN, shot noise, and thermal noise are independent noises. Therefore, the PIIN, shot noise, and thermal noise all cause independent variance in the photocurrent and thus have independent influences on system performance.

The photocurrent PIIN variance is shown in (13) and is defined as follows:

$$
\left\langle i_{\mathrm{PIIN}, \mathrm{bit}=0}^{2}\right\rangle=B_{r} I_{m, \mathrm{bit}=0}^{2} \tau,
$$

where $B_{r}$ denotes the electrical bandwidth and $\tau$ is the coherence time of the light incident on the photodiode. When the spectrum of $X_{0}(f)$ does not overlap the spectrum of
$X_{1}(f)$, the photocurrent PIIN variance of the ONU can be written as

$$
\begin{aligned}
& \left\langle i_{\text {PIIN,bit }=0}^{2}\right\rangle=B_{r} I_{m, \mathrm{bit}=0}^{2} \frac{\int_{0}^{\infty}\left[X_{0}(f)-X_{1}(f)\right]^{2} d f}{\left\{\int_{0}^{\infty}\left[X_{0}(f)-X_{1}(f)\right] d f\right\}^{2}} \\
& =B_{r} R^{2} \int_{0}^{\infty}\left[X_{0}(f)-X_{1}(f)\right]^{2} d f \\
& =B_{r} R^{2} \int_{0}^{\infty}\left[X_{0}(f)\right]^{2}+\left[X_{1}(f)\right]^{2} \\
& -2 X_{0}(f) X_{1}(f) d f=B_{r} R^{2} \int_{0}^{\infty}\left[X_{0}(f)\right]^{2} \\
& +\left[X_{1}(f)\right]^{2} d f .
\end{aligned}
$$

When the proposed system produces $X_{t}(f), t=[0,1]$ as in (10), we have $X_{t}^{2}(f), t=[0,1]$ as follows:

$$
\begin{aligned}
\int_{0}^{\infty} & X_{0}^{2}(f) d f=\frac{P_{\mathrm{sr}}^{2}}{\Delta f^{2}} \int_{0}^{\infty}\left[\sum_{p=1}^{P} \Lambda(p)\right. \\
& \left.\cdot \sum_{M^{\prime}=0}^{k-1} \sum_{O^{\prime}=0}^{k-1} \sum_{m^{\prime}=1}^{2} \sum_{q=0}^{2 k^{2}-1}\left(c_{M, O, m}(q) c_{M^{\prime}, O^{\prime}, m^{\prime}}(q)\right) F(f, q)\right]^{2} d f \\
= & \frac{P_{\mathrm{sr}}^{2}}{2 k^{2} \Delta f} \sum_{q=0}^{2 k^{2}-1}\left[\sum_{p=1}^{P} \Lambda(p)\right. \\
& \left.\cdot \sum_{M^{\prime}=0}^{k-1} \sum_{O^{\prime}=0}^{k-1} \sum_{m^{\prime}=1}^{2}\left(c_{M, O, m}(q) c_{M^{\prime}, O^{\prime}, m^{\prime}}(q)\right) F(f, q)\right]^{2} \\
& =\frac{P_{\mathrm{sr}}^{2}}{\Delta f * 2 k^{3}}\left[k+\frac{(P-1) *(k-1) * k}{2 k^{2}-2 k-1}\right]^{2}, \\
& =\frac{P_{\mathrm{sr}}^{2}}{\Delta f * 2 k^{3}}\left[\frac{(P-1) *(k-1) * k}{2 k^{2}-2 k-1}\right]^{2} \cdot \\
& \sum_{M^{\prime}=0}^{\infty} \sum_{O^{\prime}=0} \sum_{m^{\prime}=1}^{2}(f) d f=\frac{P_{\mathrm{sr}}^{2}}{\Delta f^{2}} \int_{0}^{\infty}\left[\sum_{p=1}^{P} \Lambda(p)\right. \\
& \frac{P_{\mathrm{sr}}^{2}}{2 k^{2} \Delta f} \sum_{q=0}^{2 k^{2}-1}\left[\sum_{p=1}^{P} \Lambda(p)\right. \\
& \left.\cdot \sum_{M^{\prime}=0}^{k-1} \sum_{O^{\prime}=0}^{k-1} \sum_{m^{\prime}=1}^{2} \sum_{q=0}^{2 k^{2}-1}\left(c_{M, O+1, m}(q) c_{M^{\prime}, O^{\prime}, m^{\prime}}(q)\right) F(f, q)\right]^{2} d f
\end{aligned}
$$


The PIIN has the most important effect on system performance. By substituting (17) into (16), the PIIN can be expressed as follows:

$$
\begin{aligned}
& \left\langle i_{\mathrm{PIIN}, \mathrm{bit}=0}^{2}\right\rangle \\
& =\frac{P_{\mathrm{sr}}^{2}}{\Delta f * 2 k^{3}}\left\{\left[k+\frac{(P-1) *(k-1) * k}{2 k^{2}-2 k-1}\right]^{2}\right. \\
& \left.+\left[\frac{(P-1) *(k-1) * k}{2 k^{2}-2 k-1}\right]^{2}\right\} .
\end{aligned}
$$

The proposed system uses the modified cross-correlation to produce a recovered bit with a value of 0 or 1 . Every simultaneous user produces a 0 or a 1 , which are sent with equal probability. The value of $\left\langle i_{\mathrm{PIIN}, \mathrm{bit}=1}^{2}\right\rangle$ is equal to that of $\left\langle i_{\text {PIIN,bit=0 }}^{2}\right\rangle$, so the PIIN influences the performance of the proposed system.

The shot noise also influences the photocurrent variance, and the photocurrent shot noise variance can be described as

$$
\left\langle i_{\text {shot }, \mathrm{bit}=0}^{2}\right\rangle=2 e B_{r} I_{t, \mathrm{bit}=0},
$$

where $I_{t, \mathrm{bit}=0}$ is the total photocurrent according to $I_{t, \mathrm{bit}=0}=$ $I_{\mathrm{PD} 0}+I_{\mathrm{PD} 1}$. The recovered 0 and 1 bits are sent with equal probability for every simultaneous user. The value of $\left\langle i_{\text {shot,bit=1 }}^{2}\right\rangle$ is equal to that of $\left\langle i_{\text {shot,bit=0 }}^{2}\right\rangle$. Therefore, the influence of the shot noise on system performance depends on the electronic charge and the total photocurrent.

The thermal noise arises from the effect of absolute temperature and load resistance, and the photocurrent thermal noise variance is described as follows:

$$
\left\langle i_{\text {thermal }}^{2}\right\rangle=\frac{4 K_{a} T_{n} B_{r}}{R_{L}},
$$

where $K_{a}$ is Boltzmann's constant, $T_{n}$ is the absolute temperature, and $R_{L}$ is the load resistance.

The SNR divides the square of the modified photocurrent by the photocurrent noise variances to produce the communication ability of the proposed system. Thus, the SNR is described as follows:

$$
\begin{aligned}
\mathrm{SNR}_{\mathrm{bit}=\times} & =\frac{I_{m, \mathrm{bit}=\times}^{2}}{\left\langle i_{\text {noise }}^{2}\right\rangle} \\
& =\frac{I_{m, \mathrm{bit}=\times}^{2}}{\left(\left\langle i_{\text {PIIN }, \mathrm{bit}=\times}^{2}\right\rangle+\left\langle i_{\text {shot }, \mathrm{bit}=\times}^{2}\right\rangle+\left\langle i_{\text {thermal }}^{2}\right\rangle\right)} .
\end{aligned}
$$

Because the BER uses the Gaussian approximation, it can be used to calculate the SNR. The BER can be expressed as

$$
\mathrm{BER}=\frac{\operatorname{erfc}\left(\sqrt{\left(\mathrm{SNR}_{\mathrm{bit}=0} \text { or } \mathrm{SNR}_{\mathrm{bit}=1}\right) / 2}\right)}{2},
$$

where $\operatorname{erfc}(\cdot)$ is the complementary error function as follows:

$$
\operatorname{erfc}(t)=\frac{2}{\sqrt{\pi}} \int_{t}^{\infty} \exp \left(-e^{2}\right) d e
$$

TABLE 2: Parameters used in the numerical calculation.

\begin{tabular}{lc}
\hline PD quantum efficiency & $\eta=0.6$ \\
Wavelength location & $\lambda=1.55 \mu \mathrm{m}$ \\
Data transmission rate & $2.5 \mathrm{Gbps}$ \\
Receiver noise temperature & $T_{n}=300 \mathrm{~K}$ \\
Receiver load resistor & $R_{L}=1030 \Omega$ \\
\hline
\end{tabular}

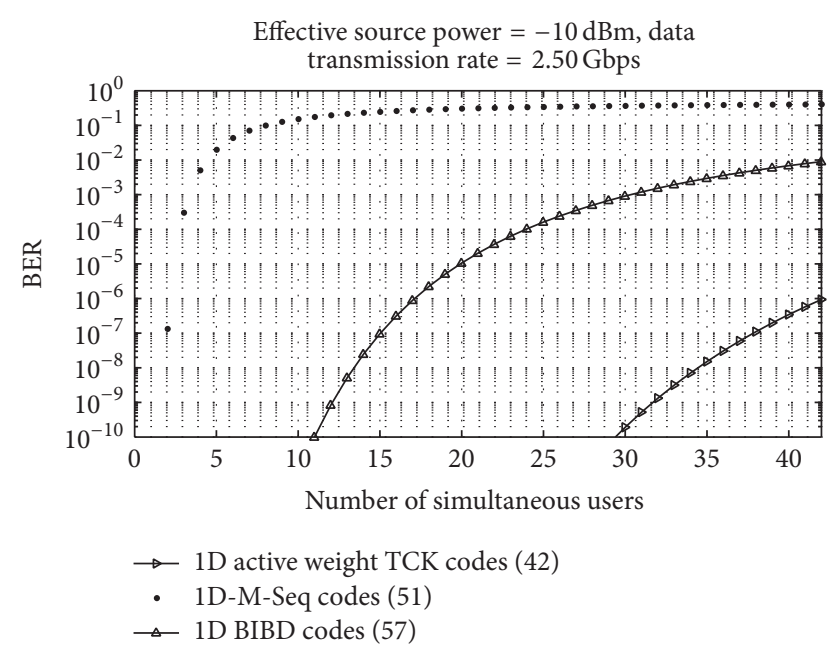

FIgURE 4: The number of simultaneous users versus BER for the SAC-OCDMA systems using 1D active weight TCK codes, 1D MSeq codes, and 1D BIBD codes.

\section{Numerical Results}

Table 2 shows the parameters used in the numerical calculation. The parameters consist of the PD quantum efficiency $\eta$, spectral width of broadband light source $\Delta \lambda$, wavelength location, data transmission rate, receiver noise temperature $T_{n}$, and receiver load resistor $R_{L}$. The parameters are calculated from the numerical results. Figure 4 shows the number of simultaneous users versus BER for the SAC-OCDMA systems using $1 \mathrm{D}$ active weight TCK codes, $1 \mathrm{D}$ M-Seq codes, and 1D BIBD codes. The proposed system and the other systems have similar code lengths. If we set the effective source power of all the systems to $-10 \mathrm{dBm}$, their data transmission rates to $2.5 \mathrm{Gbps}$, and their BERs to $10^{-9}$, we obtain 30 simultaneous users for the proposed system, one for the $1 \mathrm{D} \mathrm{M}$-Seq code system, and 11 for the $1 \mathrm{D}$ BIBD code system. The number of simultaneous users for the proposed system is larger, which improves its performance, making it superior to the other systems. Similarly, if we set effective source power to $-10 \mathrm{dBm}$, data transmission rate to $2.5 \mathrm{Gbps}$, and number of simultaneous users to 30 , the BER for the proposed system is $10^{-9}$, that for the $1 \mathrm{D} \mathrm{M}$-Seq code system is $10^{-0.5}$, and that for the $1 D$ BIBD code system is $10^{-3}$. Thus, the BER for the proposed system is lower than that for the other systems.

Figure 5 shows the data transmission rate versus BER for the SAC-OCDMA systems using 1D active weight TCK codes, 1D M-Seq codes, and 1D BIBD codes. From Figure 5, when the number of simultaneous users is 30 , the effective source power is $-10 \mathrm{dBm}$, and the $\mathrm{BER}$ is $10^{-9}$, the data 


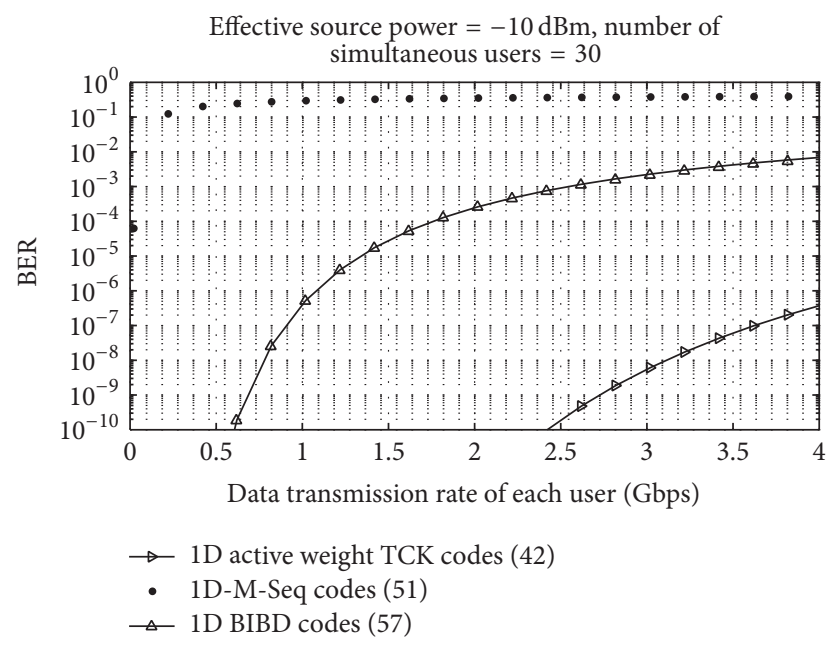

FIGURE 5: The data transmission rate versus BER for the SACOCDMA systems using 1D active weight TCK codes, 1D M-Seq codes, and ID BIBD codes.

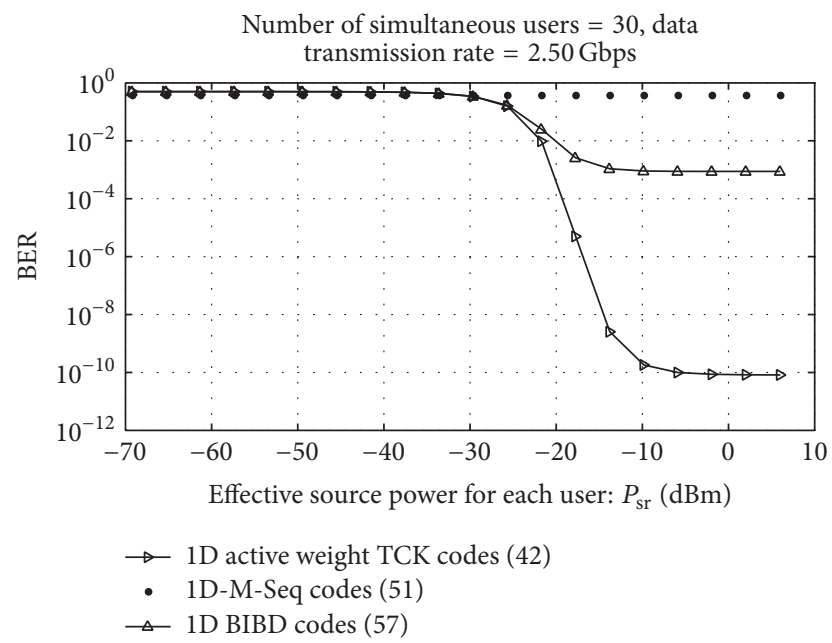

FIGURE 6: The effective source power versus BER for the SACOCDMA systems using 1D active weight TCK codes, 1D M-Seq codes, and 1D BIBD codes.

transmission rate for the proposed system is $2.5 \mathrm{Gbps}$, that for the $1 \mathrm{D}$ M-Seq code system is $0.01 \mathrm{Gbps}$, and that for the $1 \mathrm{D}$ BIBD code system is 0.74 Gbps. Thus, the data transmission rate for the proposed system is larger than that for the other systems, resulting in improved performance. When the number of simultaneous users is 30 , the effective source power is $-10 \mathrm{dBm}$, and the data transmission rate is $2.5 \mathrm{Gbps}$, the BER for the proposed system is $10^{-9}$, that for the $1 \mathrm{D} \mathrm{M}-\mathrm{Seq}$ code system is $10^{-0.5}$, and that for the $1 \mathrm{D}$ BIBD code system is $10^{-3}$. Thus, the BER for the proposed system is lower than that for the other systems.

Figure 6 shows the effective source power versus BER for the SAC-OCDMA systems using $1 \mathrm{D}$ active weight TCK codes, 1D M-Seq codes, and 1D BIBD codes. We set the number of simultaneous users to 30 users, data transmission rate to $2.5 \mathrm{Gbps}$, and $\mathrm{BER}$ to $10^{-9}$. The effective source power for the proposed system is $-10 \mathrm{dBm}$ and that for the other systems shows no crossing points. Thus, the effective source power for the proposed system is lower than that for the other systems. Finally, we set the number of simultaneous users to 30 , the data transmission rate to $2.5 \mathrm{Gbps}$, and the effective source power to $-10 \mathrm{dBm}$. The BER for the proposed system is $10^{-9}$, that for the $1 \mathrm{D} \mathrm{M}$-Seq code system is $10^{-0.5}$, and that for the $1 D$ BIBD code system is $10^{-3}$. Thus, the BER for the proposed system is lower than that for the other systems.

\section{Conclusions}

In this paper, we proposed a new family of codes for OCDMA systems named $1 \mathrm{D}$ active weight TCK codes. We used encoding and decoding transfer functions to produce the $1 \mathrm{D}$ active weight two-code keying. The proposed structure includes an optical line terminal (OLT) and optical network units (ONUs) to produce the encoding and decoding codes of the proposed OLT and ONUs, respectively. The proposed ONU uses the modified cross-correlation to calculate the recovered bits. The modified cross-correlation removes the interferences from the other simultaneous users, the MUI. The influence of the PIIN, which is noise based on the number of simultaneous users, is reduced because the proposed ONUs use the modified cross-correlation to suppress it. In the numerical results, with an effective source power of $-10 \mathrm{dBm}$, a data transmission rate of $2.5 \mathrm{Gbps}$, and a BER of $10^{-9}$, the number of simultaneous users for the proposed system using $1 \mathrm{D}$ active weight TCK codes is 30 , which is larger than that for other systems using $1 \mathrm{D}$ M-Seq codes and 1D BIBD codes. The effective source power for the proposed system using the $1 \mathrm{D}$ active weight TCK codes is $-10 \mathrm{dBm}$, which is less power than that for the other systems.

\section{Competing Interests}

The author declares no competing interests.

\section{Acknowledgments}

The author wishes to thank the High Speed Intelligent Communication (HSIC) Research Center in Chang Gung University, Taiwan, for use of the facilities and financial support.

\section{References}

[1] I. B. Djordjevic, B. Vasic, and J. Rorison, "Multi-weight unipolar codes for multimedia spectral-amplitude-coding optical CDMA systems," IEEE Communications Letters, vol. 8, no. 4, pp. 259-261, 2004.

[2] L. D. H. Sampaio, T. Abrão, B. A. Angélico, M. Lima, M. L. Proença Jr., and P. J. E. Jeszensky, "Hybrid heuristic-waterfilling game theory approach in MC-CDMA resource allocation," Applied Soft Computing Journal, vol. 12, no. 7, pp. 1902-1912, 2012.

[3] D. Kilper, K. Bergman, V. W. S. Chan, I. Monga, G. Porter, and K. Rauschenbach, "Optical networks come of age," Optics and Photonics News, vol. 25, no. 9, pp. 50-57, 2014. 
[4] W. C. Kwong, P. A. Perrier, and P. R. Prucnal, "Performance comparison of asynchronous and synchronous code-division multiple-access techniques for fiber-optic local area networks," IEEE Transactions on Communications, vol. 39, no. 11, pp. 1625$1634,1991$.

[5] D. E. Leaird, Z. Jiang, and A. M. Weiner, "Experimental investigation of security issues in OCDMA: a code-switching scheme," Electronics Letters, vol. 41, no. 14, pp. 817-819, 2005.

[6] F. R. Durand, M. L. F. Abbade, F. R. Barbosa, and E. Moschim, "Design of multi-rate optical code paths considering polarisation mode dispersion limitations," IET Communications, vol. 4, no. 2, pp. 234-239, 2010.

[7] S. Singh, R. Kaur, A. Singh, and R. S. Kaler, "Novel security enhancement technique against eavesdropper for OCDMA system using 2-D modulation format with code switching scheme," Optical Fiber Technology, vol. 22, pp. 84-89, 2015.

[8] Z. Jiang, D. S. Seo, S.-D. Yang et al., "Four-user, 2.5-Gb/s, spectrally coded OCDMA system demonstration using lowpower nonlinear processing," Journal of Lightwave Technology, vol. 23, no. 1, pp. 143-158, 2005.

[9] J.-F. Huang, K.-S. Chen, Y.-C. Lin, and C.-Y. Li, "Reconfiguring waveguide-gratings-based $\mathrm{M}$-signature codecs to enhance OCDMA network confidentiality," Optics Communications, vol. 313, pp. 223-230, 2014.

[10] M. S. Anuar, S. A. Aljunid, N. M. Saad, and S. M. Hamzah, "New design of spectral amplitude coding in OCDMA with zero cross-correlation," Optics Communications, vol. 282, no. 14, pp. 2659-2664, 2009.

[11] T. R. Raddo, A. L. Sanches, J. V. dos Reis Jr., and B.-H. V. Borges, "A new approach for evaluating the BER of a multirate, multiclass OFFH-CDMA system," IEEE Communications Letters, vol. 16, no. 2, pp. 259-261, 2012.

[12] V. Jyoti and R. S. Kaler, "Novel anti-jamming technique for OCDMA network through FWM in SOA based wavelength converter," Optical Fiber Technology, vol. 19, no. 3, pp. 259-263, 2013.

[13] A. J. Dos Santos, F. R. Durand, and T. Abrao, "Mitigation of environmental temperature variation effects in OCDMA networks using PSO power control," Journal of Optical Communications and Networking, vol. 7, no. 8, Article ID 7184838, pp. 707-717, 2015.

[14] F. R. Durand, M. S. Filho, and T. Abrão, “The effects of power control on the optical CDMA random access protocol," Optical Switching and Networking, vol. 9, no. 1, pp. 52-60, 2012.

[15] M. Tang, C. Long, and X. Guan, "Nonconvex optimization for power control in wireless CDMA networks," Wireless Personal Communications, vol. 58, no. 4, pp. 851-865, 2011.

[16] M. Noshad and K. Jamshidi, "Bounds for the BER of codes with fixed cross correlation in SAC-OCDMA systems," Journal of Lightwave Technology, vol. 29, no. 13, Article ID 5763717, pp. 1944-1950, 2011.

[17] S. Seyedzadeh, G. A. Mahdiraji, R. K. Z. Sahbudin, A. F. Abas, and S. B. A. Anas, "Experimental demonstration of variable weight SAC-OCDMA system for QoS differentiation," Optical Fiber Technology, vol. 20, no. 5, pp. 495-500, 2014.

[18] S. Mostafa, A. E. N. A. Mohamed, F. E. A. El-Samie, and A. N. Z. Rashed, "Eradication of multiple access interference using a modified multi-service code for SAC-OCDMA," Wireless Personal Communications, vol. 83, no. 2, pp. 855-872, 2015.

[19] B.-C. Yeh, "Noncoherent spectral optical code division multiple access system using one-dimensional added length codes," Optical and Quantum Electronics, vol. 48, no. 8, article 400, 2016.
[20] S. A. Aljunid, M. Ismail, A. R. Ramli, B. M. Ali, and M. K. Abdullah, "A new family of optical code sequences for spectralamplitude-coding optical CDMA systems," IEEE Photonics Technology Letters, vol. 16, no. 10, pp. 2383-2385, 2004. 


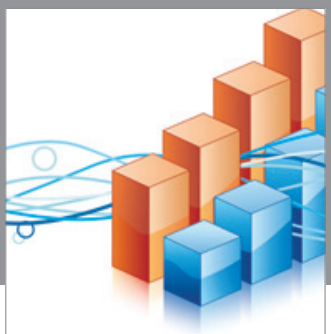

Advances in

Operations Research

vatem alat4

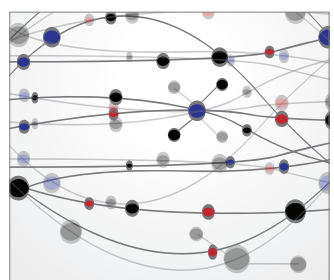

\section{The Scientific} World Journal
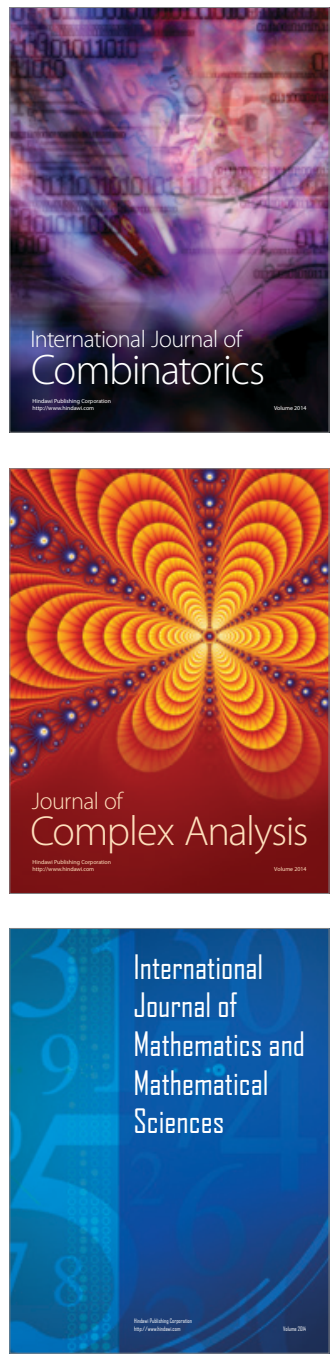
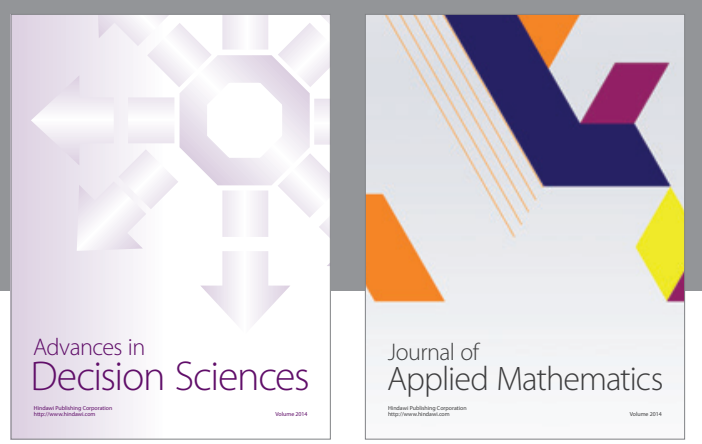

Algebra

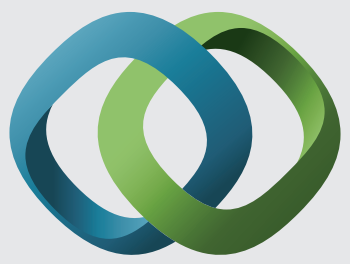

\section{Hindawi}

Submit your manuscripts at

http://www.hindawi.com
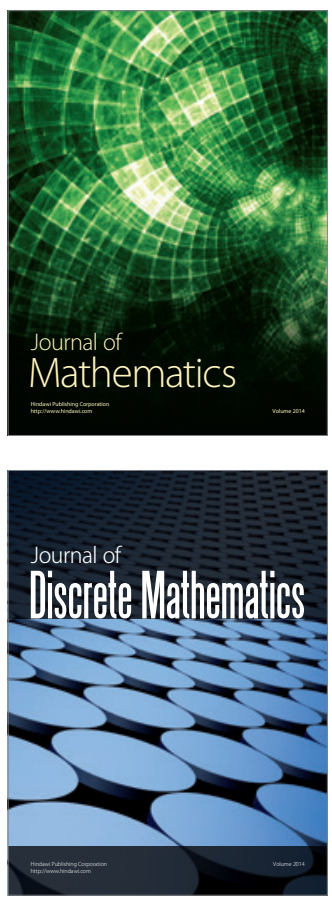

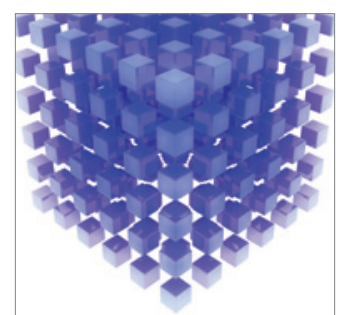

Mathematical Problems in Engineering
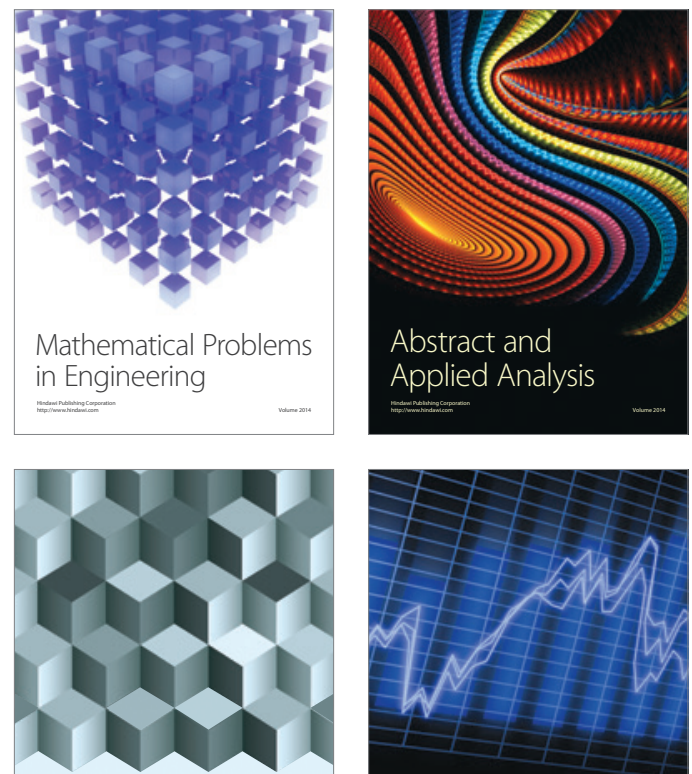

Journal of

Function Spaces

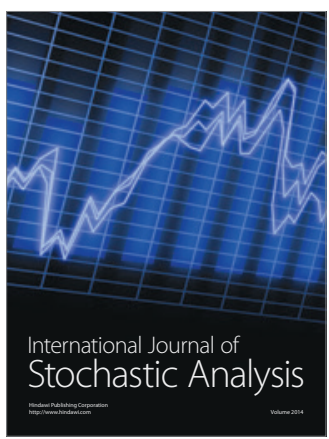

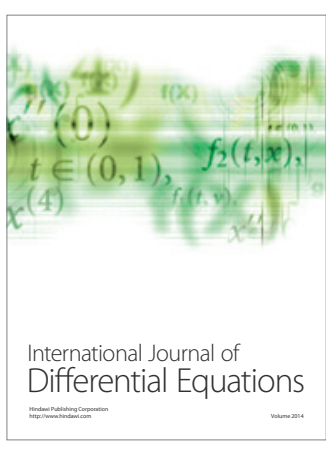
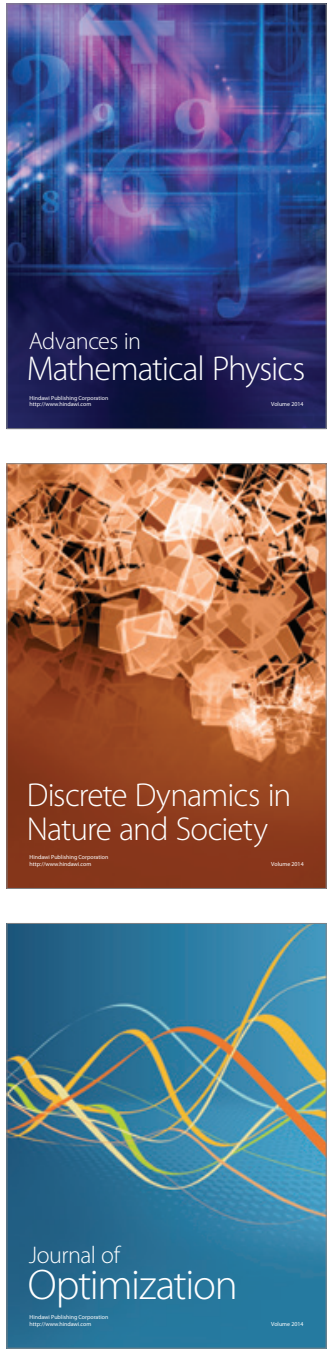